\title{
Anemia Burden, Types and Associated Risk Factors among Kenyan Human Immunodeficiency Virus-1 and Mycobacterium Tuberculosis Co-infected Injection Substance Users
}

\author{
Abonyo Collins ${ }^{1}$, Shaviya Nathan ${ }^{1}$, Budambula Valentine ${ }^{2}$, Were Tom ${ }^{1 *}$
}

\section{OPEN ACCESS}

Citation: Abonyo Collins, Shaviya Nathan; Budambula Valentine, Were Tom. Anemia Burden, Types and Associated Risk Factors among Kenyan Human Immunodeficiency Virus-1 and Mycobacterium Tuberculosis Co-infected Injection Substance Users. Ethiop J Health

Sci.2020;30(5):661.doi:http://dx.doi.org/ 10.4314/ejhs.v30i5.4

Received: May 8, 2020

Accepted: May 19, 2020

Published: September 1, 2020

Copyright: (C) 2020 Abonyo C., et al.

This is an open access article distributed under the terms of the Creative Commons

Attribution License, which permits unrestricted use, distribution, and reproduction in any medium, provided the original author and source are credited.

Funding: Nil

Competing Interests: The authors declare that this manuscript was approved by all authors in its form and that no competing interest exists.

Affiliation and Correspondence:

${ }^{1}$ School of Public Health Biomedical Sciences and Technology, Department of Medical Laboratory Sciences, Masinde Muliro University of Science and Technology, Kanya ${ }^{2}$ School of Applied and Health Sciences, Department of Environment and Health Sciences, Technical University of Mombasa, Kenya

*Email: collinsodiwuor3@gmail.com

\begin{abstract}
BACKGROUND: Although injection substance users and individuals co-infected with Human Immunodeficiency Virus-1 and Mycobacterium tuberculosis suffer marked hematologic derangements, the rates, levels, morphologic types and associated risk factors of anemia among Human immunodeficiency virus and Mycobacterium tuberculosis coinfected injection substance users has not been reported in Kenya.

METHODS: This cross-sectional study determined anemia rates, levels and morphologic types. Anemia was associated with clinical markers of disease- underweight, immunosuppression and viral load. Complete blood count, CD4 T-cell enumeration and viral load were determined via standard laboratory methods.

RESULTS: All injection substance users had higher rates of anaemia (HIV +TB+ ISUs, 79.3\%; HIV-TB+ISUs, 70.0\%; $H I V+T B-I S U S, 56.6 \%$ and $H I V-T B-I S U s, 56.2 \%)$ relative to non-ISUs (16.6\%; P<0.05). A significant proportion of $H I V+T B+I S U s$ (47.8\%) developed severe anemia than other clinical groups. The commonest morphologic type of anemia in $\mathrm{HIV}+\mathrm{TB}+\mathrm{ISU}$ s was microcytic hypochromic (43.5\%) followed by normocytic hypochromic (17.4\%) relative to the other clinical groups. HIV+TB+ ISUs with CD4 T-cells <200/uL (OR: 2.94, 95\% CI: 1.41-6.13, P=0.004) and CD4 Tcells of 200-349/uL (OR: 3.24, 95\% CI: 1.66-6.31, P=0.001) associated with higher odds of developing anemia.

CONCLUSION: This study revealed that severe anemia and microcytic hypochromic anemia are the most common erythrocytic sequelae among Human Immunodeficiency Virus-1 and Mycobacterium tuberculosis co-infected ISUs. Those with CD4 T-cells < 350/uL are utmost expected to develop anemia.

KEYWORDS: Injection substance users, Human Immunodeficiency Virus-1, Mycobacterium tuberculosis, anemia
\end{abstract}




\section{INTRODUCTION}

Injection substance use is an emerging public health problem which exacerbates the rate of HIV infection (1). It also raises the risk of HIV infected injection substance users (ISUs) developing or acquiring other comorbidities $(2,3)$. Approximately, 13 million people inject illicit substances globally; of these, about 1.7 million are HIV positive (4). HIV infected ISUs suffer other co-infections like hepatitis B virus, hepatitis $\mathrm{C}$ virus, oral candidiasis, herpes zoster and tuberculosis (TB) (3). Tuberculosis is among the most prevalent co-infections in HIV1 infected ISUs (2).

In Africa, close to 0.6 million people are injection substance users (ISUs) of whom 13.6\% are living with HIV (5). In Kenya, over 50,000 ISUs have been identified, and the number is on the increase predominantly in coastal, urban sites, peri-urban areas and cities (6). The prevalence of HIV infection in Kenyan ISUs is $\sim 18.3 \%$ with higher incidence of $18.7 \%$ mainly at the coast linked to high rates of injection substance use (7). Previous studies have reported TB to be the common co-infection in Kenyan HIV infected ISUs (8).

HIV infection is associated with marked hematological derangements including leucopenia, thrombocytopenia, immunosuppression and anemia $(9,10)$. Anemia is the most frequent hematological abnormality among HIV infected individuals more so the immunocompromised and those with higher viral load (11). Anemia can be classified as mild, moderate and severe based on the hemoglobin levels (12). Moreover, anemia can be categorized morphologically on the basis of mean corpuscular volume (MCV), mean corpuscular hemoglobin $(\mathrm{MCH})$ and mean corpuscular hemoglobin concentration (MCHC), all derived from peripheral blood films microscopically $(13,14)$. Determination of anemia types in patients is vital since it informs patient prognosis and management (15).

Anemia in HIV is further complicated by other comorbidities including injection substance use and TB (16). For instance, chronic use of methylamphetamine leads to severe aplastic anemia (17). In addition, cocaine and heroin use have been linked with iron deficiency anemia in HIV-1 infected females $(18,19)$. Furthermore, TB has been shown to cause marked hematologic derangements especially anemia in TB mono-infected patients (20). Taken together, injection substance use, HIV and TB seem to have adverse hematologic changes with severe immunosuppression and abnormal iron metabolism which can possibly lead to anemia. However, anemia rates, levels, morphologic types and related risk factors have not been characterized in Kenyan HIV-1 and TB co-infected ISUs. Therefore, we established anemia burden, levels, morphologic types and the impacting factors among HIV and TB coinfected injection substance users in Kenya.

\section{METHODS}

Study site and population: This cross-sectional study was conducted at Bomu Hospital, Mombasa County, Kenya. Upon obtaining written informed consent, 443 adults (males, $\mathrm{n}=$ 309 and females, $\mathrm{n}=134)$ were recruited, categorized into HIV+TB+ISUs $(\mathrm{n}=29)$; HIVTB+ISUs $(\mathrm{n}=20)$; HIV+TB-ISUs $(\mathrm{n}=129)$; HIV-TB-ISUs $(n=201)$ and non-ISUs $(n=64)$ with no evidence or history of injection substance use. ISUs were defined as individuals exhibiting needle scars and reporting use of any illicit injection substance from the United Nations Office on Drugs and Crime (UNODC) report (21).

Sample collection: From every non-ISU and ISU, $10 \mathrm{ml}$ of venous blood was collected. The sample was divided into EDTA and plain BD vacutainer tubes (BD, Franklin Lakes, USA). Complete blood count was conducted immediately using the fresh EDTA samples. Clotted samples in Plain BD vacutainer tubes were centrifuged at 1500 r.p.m for 10 minutes. The serum samples were obtained and used for HIV sero-testing. Sputum samples were collected in dry, clean, leak proof sterile container from ISUs with the history of prolonged coughing, fever and chest pain for more than two weeks for TB diagnosis.

HIV-1 testing: HIV-1 was tested using rapid immunochromatographic tests, Determine ${ }^{\mathrm{TM}}$ (Abbot Laboratories, Tokyo, Japan) and Unigold $^{\mathrm{TM}}$ (Trinity Biotech Plc, Bray, Ireland). Based on the Kenyan national HIV testing 
algorithm (22), ISUs who tested positive for both Determine and Unigold were confirmed infected with HIV.

TB testing: Standard sputum smears were prepared under level-3 Biosafety cabinet, heatfixed, and then covered with carbol fuchsin stain. Carbol fuchsin covered smears were then heated until vapor just begun to rise at about $60^{\circ} \mathrm{C}$ ensuring no overheating. The heated smears were allowed to remain on the slide for 5 minutes then the stain washed off with clean water. The smears were then covered with $3 \%$ $\mathrm{v} / \mathrm{v}$ acid alcohol for 5 minutes until were sufficiently decolorized (pale pink), washed well with clean water and covered with malachite green stain for 2 minutes. The stains were washed off with clean water. The back of the slides were wiped clean, and placed in a draining rack to air-dry. The stained smears were then examined microscopically, using the 100 oil immersion objective to detect the presence of mycobacterium tuberculin.

\section{Measurement of WBC, Platelets and Red Cell} Indices: $\quad \mathrm{BC}-3200$ Mindray automated Hematology analyzer (Mindray Inc, New Jersey, USA) was controlled using the normal, low and high blood controls for quality outcomes. Hematological profiles comprising total and differential WBC, RBC, Hb, HCT, MCV, MCH, MCHC, RDW, PLT, PCT and MPV were determined and assessed for derangements across, between and within study groups. Viral load determination and enumeration of CD4 Tcells were conducted as described elsewhere (23).

Ethical considerations: The study was approved by Masinde Muliro University of Science and Technology and Kenyatta University Ethical Review Committees (Ref: MMU/COR: 403012 vol2 (13) and $\mathrm{KU} / \mathrm{R} / \mathrm{COMM} / 51 / 32-4$, respectively). Written informed consent was obtained from each participant prior to enrolment into the study. HIV and TB mono and co-infected participants were referred for treatment.

Statistical analyses: Data was analyzed using GraphPad Prism 7 statistical software (GraphPad Prism version 7.00 for Windows, GraphPad Software, La Jolla California USA, www.graphpad.com.). Demographic and anthropometric characteristics (age, gender, weight, height, BMI), red blood cell counts and its indices, CD4 T-cell counts and viral load, all presented as medians and interquartile ranges (IQR), were compared across groups using Kruskal Wallis test, followed by Dunn's posthoc test for multiple comparison. Differences in the distribution of anemia, its severity and morphologic types were compared using Chisquare test. Multiple logistic regression analyses were conducted to determine associations between anemia development and BMI, CD4 Tcells and viral load in HIV+TB+ISUs. $P \leq 0.05$ was considered statistically significant.

\section{RESULTS}

Demographic and clinical profiles of the study participants: The demographic and clinical characteristics of the study participants are summarized in Table 1. A total of 443 adults (males, $\mathrm{n}=309$ and females, $\mathrm{n}=134$ ) consisting of ISUs co-infected with HIV and TB (HIV+ TB+ ISUs; $\mathrm{n}=29$ ); TB mono-infected ISUs (HIV-TB+ISUs; $\mathrm{n}=20$ ); HIV mono-infected (HIV+ TB- ISUs; $n=129$ ); uninfected (HIV- TBISUs; $n=201$ ) and non-injection substance users (HIV- TB- Non ISUs; $\mathrm{n}=64$ ) were recruited. $\mathrm{HIV}+\mathrm{TB}+$ ISUs (median, 51.0; IQR, $10 \mathrm{Kg}$ ) and HIV- TB+ ISUs (median, 49.5; IQR, 4.0 $\mathrm{Kg}$ ) had lower weight compared to the rest of the study groups $P<0.05$. Similarly, HIV+TB+ISUs, HIV+TB-ISUs, HIV-TB+ISUs and HIV-TB- ISUs had lower BMI compared to non-ISUs $(P<0.0001)$. 
Table 1: Demographic, anthropometric and drug use profiles of the study participants

\begin{tabular}{lcccccc}
\hline & & \multicolumn{5}{c}{ ISUs } \\
\cline { 3 - 5 } Characteristic & $\begin{array}{c}\text { Non-ISUs, } \\
\mathbf{n = 6 4}\end{array}$ & $\begin{array}{l}\text { HIV-TB-, } \\
\mathbf{n = 2 0 1}\end{array}$ & $\begin{array}{l}\text { HIV+TB-, } \\
\mathbf{n = 1 2 9}\end{array}$ & $\begin{array}{c}\text { HIV-TB+, } \\
\mathbf{n = 2 0}\end{array}$ & $\begin{array}{l}\text { HIV+TB+, } \\
\mathbf{n = 2 9}\end{array}$ & $\boldsymbol{P}$ \\
\hline Gender & & & & & & \\
Male & $31(48.4)$ & $188(93.5)$ & $53(41.1)$ & $18(90.0)$ & $19(65.5)$ & $<\mathbf{0 . 0 0 0 1}$ \\
Female & $33(51.6)$ & $13(6.5)$ & $76(58.9)$ & $2(10.0)$ & $10(34.5)$ & \\
Age (years) & $27.0(10.5)$ & $31.8(9.1)^{\mathrm{a}}$ & $30.6(6.5)$ & $28(5.4)$ & $30.1(6.4)$ & $\mathbf{0 . 0 1 7}$ \\
Weight $(\mathrm{kg})$ & $60.0(11)$ & $55.0(9)$ & $54.0(7)$ & $49.5(4)^{\mathrm{a}, \mathrm{c}}$ & $51.0(10)^{\mathrm{a}}$ & $<\mathbf{0 . 0 0 0 1}$ \\
Height $(\mathrm{m})$ & $1.7(0.1)$ & $1.72(0.1)^{\mathrm{a}}$ & $1.69(0.1)$ & $1.67(0.1)$ & $1.68(0.1)$ & $<\mathbf{0 . 0 0 0 1}$ \\
BMI $\left(\mathrm{kg} / \mathrm{m}^{2}\right)$ & $21.5(3.5)$ & $18.7(2.8)^{\mathrm{b}}$ & $18.8(2.1)^{\mathrm{b}}$ & $17.3(1.2)^{\mathrm{b}}$ & $18.0(2.3)^{\mathrm{b}}$ & $<\mathbf{0 . 0 0 0 1}$ \\
Drug injected & & & & & & \\
Heroin & $0(0.0)$ & $180(89.6)$ & $91(70.5)$ & $16(80.0)$ & $22(75.9)$ & \\
Cocaine & $0(0.0)$ & $18(9.0)$ & $37(28.7)$ & $2(10.0)$ & $5(17.2)$ & \\
Heroin-cocaine & $0(0.0)$ & $3(1.5)$ & $1(0.8)$ & $2(10.0)$ & $2(6.9)$ & - \\
\hline
\end{tabular}

Data are presented as medians and interquartile range for age, weight, height and BMI $\left(\mathrm{kg} / \mathrm{m}^{2}\right)$ and as number and proportion of subjects for male, female, heroin, cocaine and heroin-cocaine. BMI, body mass index; HIV, human immunodeficiency virus; TB, mycobacterium tuberculosis; HIV-TB-, HIV and TB negative injection substance users; HIV+TB-, HIV positive TB negative injection substance users; HIV-TB+, HIV negative TB positive injection substance users; HIV+TB+, HIV and TB co-infected injection substance users. Data analysis was performed using Kruskal-Wallis tests. Thereafter, post-hoc correlations for multiple comparisons were performed using the Dunn's multiple comparison tests. Statistical comparison of proportion among groups was conducted by chi-square test. The significant $P$-values are in bold. ${ }^{\mathrm{a}} P<0.05$ vs. Non-ISUs, ${ }^{\mathrm{b}} P<0.0001$ vs. Non-ISUs, ${ }^{\mathrm{c}} P<0.05$ vs. HIV+TB- ISUs for age, height and BMI, while, ${ }^{\text {a }} P<0.05$ vs. Non-ISUs for weight.

Erythrocytic measures and anemia rates, levels and morphologic types: Table 2 summarizes the erythrocytic measures and anemia rates, grading and morphologic types. Absolute red blood cell (RBC) counts differed across the clinical groups with $\mathrm{HIV}+\mathrm{TB}+$ ISUs (median, 4.4; IQR, 0.7; RBC x 10 $12 / \mathrm{L} ; P=0.001$ ) having lowest counts compared to the rest of the study groups. Similarly, hemoglobin was significantly lower in the HIV+TB+ISUs (median, 11.6; IQR, 2.8; RBC x $10^{12} / \mathrm{L} ; P<$ $0.0001)$ compared to the other groups. Also, HCT was significantly lower in HIV+TB+ISUs (median, 37.1; IQR, 8.4; HCT \%; $P=0.001$ ) compared to other clinical groups. Likewise, RDW was reduced in HIV+TB+ISUs (median, 11.9; IQR, 3.4; RDW \%; $P=0.002$ ) compared to the other groups. On the contrary, RDW was elevated in HIV+TB-ISUs compared to HIVTB-ISUs (median, 14.4; IQR, 3.0 vs. median, 14.1; IQR, 2.6; RDW \%; $P=0.004)$. Analysis revealed lower $\mathrm{MCH}$ (median, 24.7; IQR, 4.9; $P<0.05)$ in $\mathrm{HIV}+\mathrm{TB}+\mathrm{ISUs}$ compared to other groups. Also, MCHC (median, 27.9; IQR, 3.5; $P<0.05)$ was lower in HIV+TB+ISUs vis-a-vis other groups.

The World Health Organization (WHO) defines anemia in adults as hemoglobin below
$13 \mathrm{~g} / \mathrm{dL}$ in males and $12 \mathrm{~g} / \mathrm{dL}$ in females (24). Previous studies have associated HIV infection, TB and injection drug use with anemia $(18,25,26)$. We therefore analyzed anemia rates across the clinical groups. Generally, the current study found high anemia rates in all ISUs with $\mathrm{HIV}+\mathrm{TB}+$, HIV- TB+, HIV+ TB- and HIVTB- presenting with $79.3 \%, 70 \%, 56.6 \%$ and $56.2 \%$ anemia respectively. Anemia levels are classified as mild $(\mathrm{Hb}$ 10.0-12.9 $\mathrm{g} / \mathrm{dL}$ for men;10.0-11.9 g/dL for women), moderate $(\mathrm{Hb}$ 7.0-9.9 $\mathrm{g} / \mathrm{dL})$ and severe $(\mathrm{Hb}<7.0 \mathrm{~g} / \mathrm{dL})$ according to World Health Organisation (27). This study determined anemia levels across the study groups on this account. We found severe anemia in $47.8 \%$ of HIV+TB+ISUs and $8.2 \%$ of HIV+TB-ISUs. Moderate anemia was more prevalent in HIV+TB-ISUs (45.2\%), HIV-TB+ (42.9\%), HIV- TB- ISUs (30.6\%) and HIV+ TB+ ISUs $(30.5 \%)$. Mild anemia was predominant in non-ISUs $(83.4 \%)$, HIV-TBISUs (68.4\%) and HIV-TB+ISUs (57.1\%).

Clinically, erythrocytic size and intra-RBC hemoglobin are used as diagnostic markers for determining morphological types of anemia (27). As a result, we established morphological types of anemia across the clinical groups. The majority of $\mathrm{HIV}+\mathrm{TB}+\mathrm{ISUs} \quad(43.5 \%)$ had 
microcytic hypochromic anemia compared to 26.5\% HIV-TB-ISUs and 20.5\% HIV+TB-ISUs. Correspondingly, non-ISUs, HIV-TB-ISUs, HIV+TB-ISUs, HIV-TB+ISUs and

$\mathrm{HIV}+\mathrm{TB}+\mathrm{ISUs}$ presented with $16.7 \%$, $7.1 \%, 11 \%, 14.3 \%$ and $17.4 \%$ of microcytic normochromic respectively. Contrastingly, $42.9 \%$ of HIV+ TB- ISUs, $32.9 \%$ of HIV+ TBand $26.1 \%$ of HIV+ TB+ ISUs manifested with normocytic hypochromic anemia. Interestingly, $13 \%$ of HIV+ TB+ ISUs had macrocytic hyperchromic anemia suggesting $\mathrm{RBC}$ nucleus maturation defects.

Association of anaemia with BMI, CD4 - cells and viral load: Table 3 summarizes anaemia relationship with BMI, CD4 T cells and viral in
HIV and TB co-infected injection substance users. After controlling for age, gender and duration and frequency of substance injection, BMI of all categories did not indicate association with anemia development, BMI 17$18.49 \mathrm{~kg} / \mathrm{m}^{2}$ (OR: $2.60,95 \%$ CI: $0.25-26.96, P=$ 0.423 ), BMI $16-16.99 \mathrm{~kg} / \mathrm{m}^{2}$ (OR: $2.10,95 \% \mathrm{CI}$ : $0.18-24.23, P=0.551)$ and $\mathrm{BMI}<16 \mathrm{~kg} / \mathrm{m}^{2}(\mathrm{OR}:$ 2.74, 95\% CI: $0.24-30.81, P=0.413)$. Similarly, Viral load $>1000$ copies/ uL (OR: 1.05, 95\% CI: 0.26-4.21, $P=0.947$ ) showed no relationship with anaemia. Conversely, CD4 <200/uL (OR: 2.94, 95\% CI: $1.41-6.13, P=0.004)$ and CD4 of 200-349/uL (OR: 3.24, 95\% CI: 1.66-6.31, $P=$ $0.001)$ were associated with increased anemia odds.

Table 2: Erythrocytic measures and anemia rates, levels and morphologic types

\begin{tabular}{|c|c|c|c|c|c|c|}
\hline Characteristic & $\begin{array}{l}\text { Non-ISUs, } \\
\mathrm{n}=64\end{array}$ & $\begin{array}{l}\text { HIV-TB-, } \\
\text { n=201 }\end{array}$ & $\begin{array}{l}\text { HIV+TB-, } \\
\text { n=129 }\end{array}$ & $\begin{array}{l}\text { HIV-TB+, } \\
\text { n=20 }\end{array}$ & $\begin{array}{l}\text { HIV+TB+, } \\
n=29\end{array}$ & $P$ \\
\hline $\mathrm{RBC} \times 10^{12} / \mathrm{L}$ & $4.8(1.0)$ & $4.9(0.8)$ & $4.7(0.8)^{\mathrm{b}}$ & $4.9(0.6)$ & $4.4(0.7)^{\mathrm{a}}$ & 0.001 \\
\hline $\mathrm{Hb}, \mathrm{g} / \mathrm{dL}$ & $13.4(2.3)$ & $12.6(2.2)$ & $12.3(2.5)^{b}$ & $12.2(2.8)$ & $11.6(2.8)^{\mathrm{a}}$ & $<0.0001$ \\
\hline $\mathrm{HCT}, \%$ & $40.7(6.3)$ & $41.8(6.4)$ & $39.1(6.8)^{b}$ & $41.0(6.6)$ & $37.1(8.4)^{\mathrm{a}}$ & 0.001 \\
\hline RDW & $14.1(2.6)$ & $13.5(2.3)$ & $14.4(3.0)^{b}$ & $13.9(2.4)$ & $11.9(3.4)^{\mathrm{a}}$ & 0.002 \\
\hline $\mathrm{MCV}, \mathrm{fL}$ & $89.8(12.0)$ & $85.4(8.8)$ & $85.7(11.1)$ & $85.7(10.1)$ & $82.9(14.4)$ & 0.259 \\
\hline $\mathrm{MCH}, \mathrm{pg}$ & $28.7(4.7)$ & $26.4(3.8)^{\mathrm{b}}$ & $26.7(4.1)^{\mathrm{b}}$ & $26.0(3.3)$ & $24.7(4.9)^{\mathrm{a}}$ & 0.006 \\
\hline $\mathrm{MCHC}, \mathrm{g} / \mathrm{dL}$ & $32.2(3.0)$ & $30.7(3.1)^{\mathrm{b}}$ & $31.1(3.6)$ & $31.1(3.4)$ & $27.9(3.5)^{\mathrm{a}}$ & 0.013 \\
\hline Erythrocytosis, n (\%) & $1(1.6)$ & $0(0.0)$ & $0(0.0)$ & $0(0.0)$ & $0(0.0)$ & \\
\hline Polycythemia, n (\%) & $3(4.7)$ & $2(1.0)$ & $2(1.6)$ & $0(0.0)$ & $0(0.0)$ & \\
\hline Overall anaemia, $n(\%)$ & $12(16.6)$ & $98(56.2)$ & $73(56.6)$ & $14(70.0)$ & $23(79.3)$ & $<0.0001$ \\
\hline \multicolumn{7}{|l|}{ Anemia levels, n (\%) } \\
\hline Severe anemia & $0(0.0)$ & $1(1.0)$ & $6(8.2)$ & $0(0.0)$ & $11(47.8)$ & - \\
\hline Moderate anemia & $2(16.6)$ & $30(30.6)$ & $33(45.2)$ & $6(42.9)$ & $7(30.5)$ & 0.002 \\
\hline Mild anemia & $10(83.4)$ & $67(68.4)$ & $34(46.6)$ & $8(57.1)$ & $5(21.7)$ & 0.001 \\
\hline \multicolumn{7}{|l|}{ Morphologic types $n(\%)$} \\
\hline Microcytic hypochromic & $0(0.0)$ & $26(26.5)$ & $15(20.5)$ & $0(0.0)$ & $10(43.5)$ & - \\
\hline Microcytic normochromic & $2(16.7)$ & $7(7.1)$ & $8(11.0)$ & $2(14.3)$ & $4(17.4)$ & - \\
\hline Normocytic hypochromic & $2(16.7)$ & $21(21.4)$ & $19(26.0)$ & 6(42.9) & $6(26.1)$ & - \\
\hline Normocytic normochromic & $8(66.6)$ & $41(41.8)$ & $24(32.9)$ & $6(42.9)$ & $0(0.0)$ & - \\
\hline Normocytic hyperchromic & $0(0.0)$ & $2(2.1)$ & $1(1.4)$ & $0(0.0)$ & $0(0.0)$ & - \\
\hline Macrocytic hypochromic & $0(0.0)$ & $0(0.0)$ & $5(6.8)$ & $0(0.0)$ & $0(0.0)$ & - \\
\hline Macrocytic normochromic & $0(0.0)$ & $1(1.1)$ & $0(0.0)$ & $0(0.0)$ & $0(0.0)$ & - \\
\hline Macrocytic hyperchromic & $0(0.0)$ & $0(0.0)$ & $1(1.4)$ & $0(0.0)$ & $3(13.0)$ & - \\
\hline
\end{tabular}

Data are presented as medians (IQR) for RBC, Hb, HCT, RDW, MCV, MCH and MCHC and as number and proportion of subjects for, overall anemia, anemia grading and morphologic types. HIV, human immunodeficiency virus; TB, mycobacterium tuberculosis; HIV-TB-, HIV and TB negative injection substance users; HIV+TB-, HIV positive TB negative injection substance users; HIV-TB+, HIV negative TB positive injection substance users; HIV+TB+, HIV and TB co-infected injection substance users. RBC, red blood cell count; Hb, hemoglobin concentration; HCT, hematocrit; RDWcv, red blood cell distribution width; $\mathrm{MCV}$, mean corpuscular volume; $\mathrm{MCH}$, mean corpuscular hemoglobin; $\mathrm{MCHC}$, mean cell hemoglobin concentration. Data analysis was performed using Kruskal-Wallis tests. Thereafter, post-hoc correlations for multiple comparisons were performed using the Dunn's multiple comparison tests for continuous variables and chi-square test for categorical data. RBC, Hb, HCT, RDW MCH and MCHC, ${ }^{a} P<0.05$ vs. HIV-TB+, HIV+TB-, HIV-TB- and Non-ISUs; RBC, Hb, HCT and RDW, ${ }^{b} P<0.05$ vs. HIV-TB- and Non-ISUs; $\mathrm{MCH}$ and MCHC, ${ }^{\mathrm{b}} P<0.05$ vs. Non-ISUs. Significant $P$-values are shown in bold 
Table 3: Association of anaemia with BMI, CD4 T- cells and viral load

\begin{tabular}{lcccc}
\hline Characteristics & $B$ & Wald & Odds Ratios (95\% CI) & P-value \\
\hline BMI 17.00-18.49 $\left(\mathrm{kg} / \mathrm{m}^{2}\right)$ & 0.96 & 0.64 & $2.60(0.25-26.96)$ & 0.423 \\
$\quad$ 16.00-16.99( $\left.\mathrm{kg} / \mathrm{m}^{2}\right)$ & 0.74 & 0.35 & $2.10(0.18-24.23)$ & 0.551 \\
$\quad<16\left(\mathrm{~kg} / \mathrm{m}^{2}\right)$ & 1.01 & 0.67 & $2.74(0.24-30.81)$ & 0.413 \\
& & & & \\
CD4 $<200 / \mathrm{ul}$ & 1.08 & 8.29 & $2.94(1.41-6.13)$ & $\mathbf{0 . 0 0 4}$ \\
$200-349 / \mathrm{ul}$ & 1.18 & 11.93 & $3.24(1.66-6.31)$ & $\mathbf{0 . 0 0 1}$ \\
$350-499 / \mathrm{ul}$ & 0.23 & 0.55 & $1.25(0.68-2.31)$ & 0.459 \\
& & & & \\
Viral Load $>1000 \mathrm{cps} / \mathrm{ul}$ & 0.47 & 0.01 & $1.048(0.261-4.215)$ & 0.947 \\
\hline
\end{tabular}

Anaemia relationship with BMI, CD4 T - cells and viral load after controlling for age, gender, duration and frequency of substance injection. BMI $\left(\mathrm{kg} / \mathrm{m}^{2}\right)$ categorized according to WHO (49) while CD4 T-cells categorized as per (50).

\section{DISCUSSION}

This study focused on the rates, levels, morphologic types and related risk factors of anemia in ISUs. We compared distribution in $\mathrm{HIV}+\mathrm{TB}+$ ISUs, HIV-TB + ISUs, HIV + TBISUs, HIV- TB- ISUs and non- ISUs with the main outcome group being $\mathrm{HIV}+\mathrm{TB}+$ ISUs. RBCs, in their normal quantities and morphology, transport and deliver oxygen to all human body tissues (28). Derangements in RBC indices impair the body's physiological and homeostatic roles $(29,30)$. This further leads to tissue hypoxia (31), disease progression, poor prognosis hence shortened life span (32). While studies have described levels, rates, morphologic types and associated risk factors of anemia in HIV-1 infected individuals, TB and HIV-TB coinfected individuals $(26,33,34)$, little is known about anemia in the context of HIV-1 and TB co-infected Kenyan injection substance users.

We found high anemia rates and levels in all diseased clinical groups. These findings imply that injection substance use, HIV-1 and TB appear to modulate anemia. Similarly, other studies have reported anemia independently in the context of injection substance use, HIV-1 and TB. For instance, injection substance use has been shown as an independent risk factor associated with iron deficiency anemia (35). Also, HIV-1 infection is associated with low hemoglobin levels (36). Likewise, several recent studies have reported anemia in TB infected patients (34). However, a combination of all these possible anemia etiologies has not been studied in the milieu of Kenyan ISUs.

Consequently, the current study found very high rates and levels of anemia in HIV and TB co-infected ISUs with a majority presenting with severe anemia. A case study in a heroin addicted injection drug user suggested that hitting the groinal femoral artery with a syringe instead of the femoral vein could be causing iron deficiency anemia in the patient (37). Additionally, Cocaine use has been linked with iron build up in the brain hence depleting iron supply for hemoglobin synthesis (38). Like other studies, we found ISUs to have low BMI suggesting malnutrition among injection substance users which is associated with iron deficiency anemia. HIV has been suggested to directly infect erythroid progenitor cells causing anemia $(39,40)$. Moreover, HIV derived proteins and cytokines have been proposed as inhibitors of hematopoietic cells in the bone marrow leading to anemia $(41,42)$. The most plausible mechanism implicated in anemia in pulmonary TB is chronic inflammation that leads to malabsorption of iron (34). Consequently, there is a possibility of an amalgamation of these mechanisms as well as malnutrition resulting in high anemia rates and levels among HIV+ TB+ ISUs.

Normocytic normochromic and microcytic hypochromic anemia were the most common morphologic types in ISUs mainly, HIV- TB-, $\mathrm{HIV}+\mathrm{TB}-$, HIV- TB+. This suggests that TB infection, HIV disease, cocaine and heroin use 
play key roles in pathophysiology of these anemia types. This finding is corroborated with previous studies which have reported the majority of TB and HIV patients with normocytic normochromic and microcytic hypochromic anemias $(11,43,44)$. Furthermore, heroin and cocaine have been shown to disrupt iron regulation and depress developmental potentials of hematopoietic stem cells $(17,45,46)$. Contrarily, we found dominance of microcytic hypochromic anemia in $\mathrm{HIV}+\mathrm{TB}+$ ISUs $(43.8 \%)$. Consistent with lower BMI suggesting malnutrition, altered iron metabolism, reduced production of erythropoietin and suppressed bone marrow activity could be the probable explanations for this phenomenon.

Nonetheless, injection substance users with CD4 $<350$ cells/uL were highly associated with anaemia, a finding in agreement with previous studies which have reported $\mathrm{CD} 4<350 \mathrm{uL}$ as one of the major predictors of anaemia among injection substance users (47). This could suggest suppressed erythropoietic activity by heroin or cocaine, viral progression and aggressive invasion by opportunistic tuberculosis. Thus, HIV, its virulent proteins, $\mathrm{TB}$, heroin, cocaine and proinflammatory cytokines including IL-1, IL-6, TNF- $\alpha$ altogether could be responsible for anemia severity among HIV+ TB+ ISUs (48).

In conclusion, these results indicate that weight loss, severe anemia and microcytic hypochromic anemia are the most common anthropometric and erythrocytic deviations among HIV and TB co-infected ISUs with CD4 T-cells $<350 / \mathrm{uL}$ in the Kenyan Coast. This is probably due to micronutrient deficiencies, chronic inflammation and direct or indirect effects of HIV, TB and illicit substance injection. The management of HIV and TB coinfected ISUs in our view should incorporate iron, folate and vitamin $\mathrm{B}_{12}$ levels determination. In addition, cases with insufficiencient iron or vitamins be given supplements to improve haematologic recovery.

Definition of ISUs was based on selfreported definition. Also, no toxicological analysis was performed on urine or serum samples to identify and quantify substances used including their metabolites. Moreover, ferritin levels and reticulocyte counts were not done to reinforce diagnosis of iron deficiency anemia suggested by microcytic hypochromic cells. Likewise, bone marrow aspirations and biopsies were not examined to give more information on marrow hematopoietic potentials among ISUs.

\section{ACKNOWLEDGEMENTS}

We express our gratitude to study participants for making this study possible. We also thank the staff of Bomu Hospital for their endless support throughout the study period.

\section{REFERENCES}

1. Bruce RD, Altice FL. Clinical Care of the HIV-Infected Drug User. Infect Dis Clin North Am. 2007 Mar;21(1):149-ix.

2. Deiss RG, Rodwell TC, Garfein RS. Tuberculosis and Drug Use: Review and Update. Clin Infect Dis Off Publ Infect Dis Soc Am [Internet]. 2009 Jan 1 [cited 2018 May 28];48(1).

3. Solomon SS, Hawcroft CS, Narasimhan P, Subbaraman R, Srikrishnan AK, Cecelia AJ, et al. Comorbidities among HIV-infected injection drug users in Chennai, India. Indian J Med Res. 2008 May;127(5):44752.

4. WHO. People who inject drugs [Internet]. 2019 [cited 2019 Jul 22].

5. Raguin G, Lepretre A, Ba I, Ndoye I, Toufik A, Brucker G, et al. Drug use and HIV in West Africa: a neglected epidemic. Trop Med Int Health TM IH. 2011 Sep;16(9):1131-3.

6. NACC. Kenya AIDS Indicator Survey [Internet]. National AIDS Control Council. 2012 [cited 2019 Jul 22].

7. Budambula V, Musumba FO, Webale MK, Kahiga TM, Ongecha-Owuor F, Kiarie JN, et al. HIV-1 protease inhibitor drug resistance in Kenyan antiretroviral treatment-naive and -experienced injection drug users and non-drug users. AIDS Res Ther. 2015; 12:27.

8. WHO. TB and HIV [Internet]. WHO. 2019 [cited 2019 Jul 22]. 
9. Geletaw T, Tadesse MZ, Demisse AG. Hematologic abnormalities and associated factors among HIV infected children preand post-antiretroviral treatment, North West Ethiopia. J Blood Med. 2017 Aug 4;8:99-105.

10. Vishnu P, Aboulafia DM. Haematological manifestations of human immune deficiency virus infection. $\mathrm{Br} J$ Haematol. 2015;171(5):695-709.

11. Parinitha S, Kulkarni M. Haematological changes in HIV infection with correlation to CD4 cell count. Australas Med J. 2012;5(3):157-62.

12. Mengesha MB, Dadi GB. Prevalence of anemia among adults at Hawassa University referral hospital, Southern Ethiopia. BMC Hematol. 2019;19:1.

13. Elgari MM, Khabour OF, Elhag HAEH, Muddathir ARM. Hematological indices of end-stage chronic renal failure patients in Sudan: With or without iron supplements. Pak J Pharm Sci. 2019 Mar;32(2 (Supplementary)):765-8.

14. Sarma PR. Red Cell Indices. In: Walker HK, Hall WD, Hurst JW, editors. Clinical Methods: The History, Physical, and Laboratory Examinations [Internet]. 3rd ed. Boston: Butterworths; 1990 [cited 2019 Jul 22].

15. Turner J, Badireddy M. Anemia. In: StatPearls [Internet]. Treasure Island (FL): StatPearls Publishing; 2019 [cited 2019 Jul 22].

16. Masaisa F, Gahutu JB, Mukiibi J, Delanghe J, Philippé J. Anemia in Human Immunodeficiency Virus-Infected and Uninfected Women in Rwanda. Am J Trop Med Hyg. 2011 Mar 4;84(3):456-60.

17. Nixon CC, Schwartz BH, Dixit D, Zack JA, Vatakis DN. Cocaine exposure impairs multilineage hematopoiesis of human hematopoietic progenitor cells mediated by the sigma-1 receptor [corrected]. Sci Rep. 2015 Mar 2;5:8670.

18. Dancheck B, Tang AM, Thomas AM, Smit E, Vlahov D, Semba RD. Injection drug use is an independent risk factor for iron deficiency and iron deficiency anemia among HIV-seropositive and HIV- seronegative women. $J$ Acquir Immune Defic Syndr 1999. 2005 Oct 1;40(2):198201.

19. Semba RD, Shah N, Strathdee SA, Vlahov D. High prevalence of iron deficiency and anemia among female injection drug users with and without HIV infection. $J$ Acquir Immune Defic Syndr 1999. 2002 Feb $1 ; 29(2): 142-4$.

20. Malvoisin E, Makhloufi D, Livrozet J-M. Serum hepcidin levels in women infected with HIV-1 under antiviral therapy. $J$ Med Virol. 2014 Oct 1;86(10):1656-60.

21. UNODC. World Drug Report [Internet]. 2014 [cited 2018 Apr 23].

22. 2NASCOP. HIV Testing Services [Internet]. NASCOP. 2019 [cited 2019 Jul 27].

23. Were T, Wesongah JO, Munde E, Ouma C, Kahiga TM, Ongecha-Owuor F, et al. Clinical chemistry profiles in injection heroin users from Coastal Region, Kenya. BMC Clin Pathol. 2014;14:32.

24. 2WHO. Anemia [Internet]. WHO. 2019 [cited 2019 Jul 25].

25. Hella J, Cercamondi CI, Mhimbira F, Sasamalo M, Stoffel N, Zwahlen M, et al. Anemia in tuberculosis cases and household controls from Tanzania: Contribution of disease, coinfections, and the role of hepcidin. PLOS ONE. 2018 Apr 20 [cited 2019 Jul 25];13(4).

26. Martin C, Poudel-Tandukar K, Poudel KC. HIV Symptom Burden and Anemia among HIV-Positive Individuals: Cross-Sectional Results of a Community-Based Positive Living with HIV (POLH) Study in Nepal. PLoS ONE. 2014 Dec 31 [cited 2018 Apr $10] ; 9(12)$.

27. WHO. Haemoglobin concentrations for the diagnosis of anaemia and assessment of severity. WHO. 2019 [cited 2019 Jul 25].

28. Focosi D, Amabile G. Induced Pluripotent Stem Cell-Derived Red Blood Cells and Platelet Concentrates: From Bench to Bedside. Cells. 2017 Dec 27;7(1).

29. Kozuki N, Lee AC, Katz J. Moderate to Severe, but Not Mild, Maternal Anemia Is Associated with Increased Risk of Smallfor-Gestational-Age Outcomes. J Nutr. 2012 Feb 1;142(2):358-62. 
30. Lacroix J, Hébert P, Fergusson D, Tinmouth A, Blajchman MA, Callum J, et al. The Age of Blood Evaluation (ABLE) randomized controlled trial: study design. Transfus Med Rev. $2011 \mathrm{Jul} ; 25(3): 197-205$.

31. Abrams DI, Steinhart C, Frascino R. Epoetin alfa therapy for anaemia in HIV-infected patients: impact on quality of life. Int $J$ STD AIDS. 2000 Oct;11(10):659-65.

32. Brunetta DM, Santis D, Cunha G, Vilar FC, Brandão RA, Muniz RZ de A, et al. Hematological particularities and coinfections in injected drug users with AIDS. Braz J Infect Dis. 2013 Dec;17(6):654-6.

33. Semba RD, Shah N, Strathdee SA, Vlahov D. High prevalence of iron deficiency and anemia among female injection drug users with and without HIV infection. $J$ Acquir Immune Defic Syndr 1999. 2002 Feb 1;29(2):142-4.

34. Gil-Santana L, Cruz LAB, Arriaga MB, Miranda PFC, Fukutani KF, Silveira-Mattos PS, et al. Tuberculosis-associated anemia is linked to a distinct inflammatory profile that persists after initiation of antitubercular therapy. Sci Rep. 2019 Feb 4;9(1):1381.

35. Dancheck B, Tang AM, Thomas AM, Smit E, Vlahov D, Semba RD. Injection drug use is an independent risk factor for iron deficiency and iron deficiency anemia among HIV-seropositive and HIVseronegative women. $J$ Acquir Immune Defic Syndr 1999. 2005 Oct 1;40(2):198201.

36. Panwar A, Sharma SC, Kumar S, Sharma A. A study of anemia in human immunodeficiency virus patients: Estimating the prevalence, analyzing the causative effect of nutritional deficiencies, and correlating the degree of severity with CD4 cell counts. Med J Dr Patil Univ. 2016 May $1 ; 9(3): 312$

37. Younis N, Casson IF. An unusual cause of iron deficiency anaemia in an intravenous drug user. Hosp Med Lond Engl 1998. 2000 Jan;61(1):62-3.

38. Nelson S, Lerner E, Needlman R, Salvator A, Singer LT. Cocaine, anemia, and neurodevelopmental outcomes in children: a longitudinal study. J Dev Behav Pediatr JDBP. 2004 Feb;25(1):1-9.

39. Bagnara GP, Zauli G, Giovannini M, Re MC, Furlini G, La Placa M. Early loss of circulating hemopoietic progenitors in HIV1-infected subjects. Exp Hematol. 1990 Jun; 18(5):426-30.

40. Tsukamoto T. HIV Impacts CD34+ Progenitors Involved in T-Cell Differentiation During Coculture With Mouse Stromal OP9-DL1 Cells. Front Immunol. 2019 [cited 2019 Jul 26];10.

41. Cavallaro F, Duca L, Pisani LF, Rigolini R, Spina L, Tontini GE, et al. Anti-TNFMediated Modulation of Prohepcidin Improves Iron Availability in Inflammatory Bowel Disease, in an IL-6-Mediated Fashion. Canadian Journal of Gastroenterology and Hepatology. 2017 [cited 2019 Jul 26].

42. Schmidt IHE, Gildhorn C, Böning MAL, Kulow VA, Steinmetz I, Bast A. Burkholderia pseudomallei modulates host iron homeostasis to facilitate iron availability and intracellular survival. PLoS Negl Trop Dis. 2018 Jan 12;12(1):e0006096.

43. Monjur F, Rizwan F. A Cross-sectional Study of Morphological Types of Anemia in Pulmonary Tuberculosis Patient and Associated Risk Indicators in a Selected Hospital of Dhaka City, Bangladesh. In 2014.

44. Saha D, Kini JR, Subramaniam R. A study of the hematological profile of human immunodeficiency virus positive patients in coastal South Indian region. J Med Sci. 2015 Jan 9;35(5):190.

45. Haghpanah T, Afarinesh M, Divsalar K. A Review on Hematological Factors in Opioid-Dependent People (Opium and Heroin) after the Withdrawal Period. Addict Health. 2010;2(1-2):9-16.

46. Ersche KD, Acosta-Cabronero J, Jones PS, Ziauddeen H, Swelm RPL van, Laarakkers $\mathrm{CMM}$, et al. Disrupted iron regulation in the brain and periphery in cocaine addiction. Transl Psychiatry. 2017 Feb;7(2):e1040.

47. Semba RD, Shah N, Vlahov D. Risk factors and cumulative incidence of anaemia among 
HIV-infected injection drug users. Int J STD AIDS. 2002 Feb;13(2):119-23.

48. Enawgaw B, Alem M, Addis Z, Melku M. Determination of hematological and immunological parameters among HIV positive patients taking highly active antiretroviral treatment and treatment naïve in the antiretroviral therapy clinic of Gondar University Hospital, Gondar, Northwest
Ethiopia: a comparative cross-sectional study. BMC Hematol. 2014 Mar 25;14:8.

49. WHO. Physical status: the use and interpretation of anthropometry. World Health Organization; 2020 [cited 2020 Apr 24].

50. 5WHO. Interim WHO clinical staging of HIV/AIDS and HIV/AIDS case definitions for surveillance. WHO. 2005 [cited 2019 Nov 26]. 